\title{
Abordagem fisioterapêutica da hiperatividade detrusora na Esclerose Múltipla: revisão de literatura
}

\author{
Physical therapeutic approach on detrusor overactivity in Multiple Sclerosis: literature review
}

Josiane Lopes ${ }^{1}$, Damacio Ramón Kaimen-Maciel ${ }^{2}$

\section{RESUMO}

Introdução. A hiperatividade detrusora neurogênica (HD) é a disfunção vesical mais comum em indivíduos com esclerose múltipla (EM) sendo acompanhada do sintoma de urge-incontinência (UrI) em $83 \%$ dos casos. O tratamento padráo é o farmacológico, que apresenta evidência bem estabelecida, ao contrário do tratamento fisioterapêutico. Objetivo. Discutir os procedimentos fisioterapêuticos na HD decorrente da EM. Método. Foi realizada uma revisão através de pesquisa nas bases de dados Cochrane Library, Ibecs Lilacs, Medline, PEDro e Scielo utilizando os descritores: acupuntura, bexiga neurogênica, biofeedback, disfunção vesical, eletroacupuntura, eletroestimulação, esclerose múltipla, exercícios físicos, exercícios de Kegel, fisioterapia, hiperatividade detrusora, incontinência urinária, reabilitaçáo do assoalho pélvico, terapia comportamental e treinamento físico, urge-incontinência. Resultados. Foram encontrados 26 artigos e selecionados $10 \mathrm{com}$ procedimentos indicados devido a correlaçáo estatística com a melhora do quadro de HD e UrI $(\mathrm{p} \leq 0,05)$. Dentre os artigos, 6 evidenciaram a aplicaçáo da forma isolada da terapia miofascial, exercícios de Kegel e eletroestimulação. O restante combinou exercícios de Kegel, eletroestimulação, biofeedback eletromiográfico e orientaçóes. Conclusáo. Nenhum procedimento fisioterapêutico foi evidenciado como essencial no manejo da HD/ UrI em indivíduos com EM, sendo observada que a abordagem combinada dos procedimentos apresentou os níveis de melhor evidência nesta disfunção.

Unitermos. Esclerose Múltipla, Detrusor Hiperativo, Fisioterapia, Incontinência Urinária de Urgência.

Citaçáo. Lopes J, Kaimen-Maciel DR. Abordagem fisioterapêutica da hiperatividade detrusora na Esclerose Múltipla: revisão de literatura.

\begin{abstract}
Introduction. The neurogenic detrusor overactivity (DO) is an usual bladder disorder in subjects with multiple Sclerosis (MS) showing urinary incontinence, Urge (Ur) in $83 \%$ of cases. The standard treatment is pharmacological with well established evidence, contrary physiotherapy. Objective. to discuss the physiotherapeutic procedures in DO due to MS. Method. A review was conducted by searching the databases Cochrane Library, Ibecs, Lilacs, Medline, PEDro, and Scielo using the keywords: acupuncture, neurogenic bladder, biofeedback, bladder dysfunction, electroacupuncture, electro-stimulation, multiple sclerosis, physical exercises, Kegel exercises, physiotherapy, detrusor overactivity, urinary incontinence, pelvic floor rehabilitation, behavioral therapy and physical training, and urge incontinence. Results. There were found 26 articles and selected 10 with procedures indicated due to a statistical correlation with improvement of symptoms of DO and $\mathrm{Ur}(\mathrm{p} \leq 0.05)$. Among the articles, 6 demonstrated the application as unique way: myofascial therapy, Kegel exercises and electrical stimulation. The remaining combined Kegel exercises, electrical stimulation, electromyographic biofeedback, and orientations. Conclusion. None of physiotherapeutic procedures was seen as essential in the management of DO/ Ur in subjects with MS and it was observed that the combined approach procedures showed the levels of best evidence in this disorder.
\end{abstract}

Keywords. Multiple sclerosis, Urinary Bladder Overactivity, Physical Therapy, Urinary Incontinence, Urge.

Citation. Lopes J, Kaimen-Maciel DR. Physical therapeutic approach on detrusor overactivity in Multiple Sclerosis: literature review.
Trabalho realizado na Universidade Estadual de Londrina (UEL), Londrina-PR, Brasil.

1.Fisioterapeuta, Mestre em Medicina e Ciências da Saúde (UEL), Doutoranda em Ciências da Saúde (UEL), Londrina-PR, Brasil.

2.Neurologista, Pós-doutor em Neurologia, Professor associado do departamento de clínica médica da UEL, Londrina-PR, Brasil.
Endereço para correspondência: Josiane Lopes Av. Inglaterra, 155 CEP 86046-000, Londrina-PR, Brasil. E-mail: josianelopes@yahoo.com.br 


\section{INTRODUÇÃO}

A Esclerose Múltipla (EM) é uma doença desmielinizante, inflamatória, degenerativa e crônica da substância branca do sistema nervoso central de etiologia idiopática que acomete principalmente adultos jovens sendo mais prevalente no sexo feminino e entre brancos de origem norte-européia ${ }^{1,2}$. Apresenta evolução progressiva e imprevisível com manifestaçôes multiformes de déficits funcionais que podem decorrer da lesão a qualquer parte do neuroeixo ${ }^{1}$. Dentre estes déficits, as alteraçôes miccionais resultantes das disfunçôes vésico-esfincterianas estão presentes em até $80 \%$ dos casos podendo ser sintoma inaugural em 10 a 35\% dos indivíduos com $\mathrm{EM}^{3}$.

As disfunçóes vésico-esfincterianas associadas à EM variam amplamente. Podem apresentar-se num espectro desde hipocontratilidade detrusora e retenção urinária até hiperatividade do músculo detrusor (HD) e dissinergia vésico-esfincteriana ${ }^{4}$. Dados clínicos demonstram que $96 \%$ dos pacientes com EM apresentam anormalidades no músculo detrusor com predomínio da condição de HD e no funcionamento esfincteriano, ambos relacionados à desmielinização do trato córtico-espinhal e/ou retículo-espinhal 5 .

$\mathrm{Na}$ EM, a bexiga hiperativa é o tipo mais comum. Segundo a Sociedade Internacional de Continência, este tipo pode ser classificado em hiperatividade detrusora neurogênica (HD), quando a causa é neurológica ${ }^{6}$. Nesta condição o enchimento vesical desencadeia contraçóes não inibidas, que passam a ser interpretadas como urgência miccional, tornando a capacidade vesical reduzida ${ }^{7}$. Os sintomas da bexiga hiperativa são variados e em 90\% dos casos associam-se à $\mathrm{HD}^{8}$. Esta síndrome possui um diagnóstico clínico caracterizado por urgência miccional com urge-incontinência (UrI), usualmente acompanhada de noctúria e de aumento da frequência urinária9 ${ }^{9}$. Associada à EM, os indivíduos relatam sintomas urinários irritativos, incontinência urinária (IU), urgência e frequência, sendo a queixa de UrI a mais relatada acometendo $83 \%$ dos $\operatorname{casos}^{10}$.

Considerando a evolução da EM, é observada com maior frequência a presença desses sintomas urinários em casos com mais de quatro anos de diagnóstico ${ }^{11}$. Estudos relatam que $75 \%$ a $90 \%$ dos pacientes com EM vão apresentar UrI em algum período da doença, sugerindo que este sintoma está diretamente ligado à evolução da $\mathrm{EM}^{12,13}$. Os portadores também podem sofrer de sintomas debilitantes do trato urinário, como frequentes infecçôes do trato urinário, danos ao trato urinário superior e quadros de hidronefrose, sendo mais encontradas nos quadros de dissinergia vésico-esfincteriana ${ }^{14}$.

As disfunçôes vesicais causam repercussão física, psíquica e social com impacto negativo na qualidade de vida dos portadores de EM devido principalmente pelo sofrimento por privação social ${ }^{14}$. Há relatos de que as disfunçôes miccionais na EM constituem o terceiro fator mais importante de limitação produtiva, perdendo apenas para a presença de espasticidade e fadiga ${ }^{15}$.

A frequência e repercussão da HD na EM revela a necessidade de conhecimentos sobre as formas de manejo e tratamento. $\mathrm{O}$ tratamento deve ser guiado para promover o esvaziamento vesical completo, preservar o bom funcionamento renal, evitar complicaçóes do tracto urinário inferior e permitir um esvaziamento funcional. As principais modalidades terapêuticas são o tratamento farmacológico e as terapias conservadoras, dentre elas a fisioterapia. Como última alternativa, surge a abordagem cirúrgica ${ }^{16}$. Medidas farmacológicas como o uso de anticolinérgicos, toxina botulínica ou o emprego da neuromodulação apresentam eficácia bem estabelecida em manter baixa as pressóes de armazenamento e esvaziamento devido ao relaxamento do detrusor ${ }^{14,17}$. Já em situaçóes em que a HD vem acompanhada da incompetência esfincteriana demonstrada pela UrI, o tratamento fisioterapêutico pode ser indicado ${ }^{18}$.

Os procedimentos fisioterapêuticos integram o grupo de recursos conservadores da abordagem da terapia comportamental que auxiliam no manejo da HD e seu quadro clínico, contribuindo para a reeducação vesical e do assoalho pélvico ${ }^{18}$. Entretanto, são pouco relatados os níveis de evidência quanto aos benefícios terapêuticos e diretrizes da utilização da fisioterapia no tratamento da HD na $\mathrm{EM}^{19}$.

Assim, o objetivo desta revisão foi discutir os estudos sobre a abordagem fisioterapêutica na HD associada à EM identificando as técnicas e recursos fisioterapêuticos segundo a evidência estatística dos procedimentos na melhora sintomática dos quadros de HD e seu sintoma mais frequente, a UrI. 


\section{MÉTODO}

O estudo propôs uma revisão bibliográfica sobre procedimentos fisioterapêuticos na HD e seu sintoma mais frequente, a UrI, associada à EM. Foi realizada uma pesquisa computadorizada nas bases de dados Cochrane Library, Ibecs, Lilacs, Medline, PEDro, e Scielo tendo como enfoque a problemática: Qual(is) o(s) procedimento(s) fisioterapêutico(s) e sua(s) diretrizes para a abordagem da HD e a UrI resultante em indivíduos com EM?

Foram utilizados os descritores de procedimentos fisioterapêuticos: acupuntura, biofeedback, eletroacupuntura, eletroestimulação, exercícios físicos, exercícios de Kegel, fisioterapia, reabilitação do assoalho pélvico, terapia comportamental e treinamento físico. Os descritores de disfunção: bexiga neurogênica, disfunção vesical, hiperatividade detrusora, incontinência urinária, urge-incontinência. E o descritor da doença: esclerose múltipla. Estes descritores foram submetidos à técnica de cruzamento por meio do operador booleano "e" sempre utilizando um descritor de procedimento fisioterapêutico, um descritor de disfunção e o descritor "esclerose múltipla". Todos os descritores de procedimentos fisioterapêuticos e de disfunção foram cruzados entre si.

A seleção dos artigos considerou os critérios de inclusão: estudos em humanos, adultos, correlação da HD e/ ou UrI com a atuação fisioterapêutica. Foram excluídos os artigos que enfocam o tratamento fisioterapêutico de outros sintomas da EM além do HD e UrI, estudos com dados não conclusivos, revisões de literatura, editoriais e cartas.

A análise e discussão dos dados foram realizadas apresentando as técnicas/ recursos e diretrizes que contemplam os procedimentos fisioterapêuticos específicos para o tratamento da HD e/ ou UrI resultante na EM, nas formas isolada e combinada. Foi considerado como procedimento fisioterapêutico indicado ao tratamento aquele que apresenta correlação estatística com valor de $\mathrm{p}$ $\leq 0,05$ com a melhora da HD e o quadro de UrI, apresentando, portanto, evidência estatisticamente significativa de que é uma intervenção com efeito terapêutico.

\section{RESULTADOS}

Foram encontrados 26 artigos sendo selecionados 10 (Quadro 1) que evidenciam resultados aplicáveis na prática fisioterapêutica para o manejo da HD em indivíduos com EM. Dentre os estudos, 6 apresentaram aplicação isolada das técnicas fisioterapêuticas: Eletroestimulaçấo ${ }^{20-23}$, Terapia manual ${ }^{24}$ e Cinesioterapia ${ }^{25}$. Os demais combinaram técnicas cinesioterapêuticas, eletroterapêuticas e orientaçôes (Quadro 1).

O emprego da eletroestimulação foi realizado de forma isolada e combinada em protocolos com cinesioterapia e biofeedback. As modalidades utilizadas foram a eletroestimulação neuromuscular (EENM) periférica no nervo tibial com aplicação transcutânea ${ }^{20,21}$ perineometria vaginal ${ }^{22,26}$ e aplicação no dermátomo sacral ${ }^{23}$. Os protocolos dos estudos demonstraram utilização do máximo estímulo intermitente tolerado sem dor (mínimo de 15 miliampéres), com razão temporal estímulo/descanso mínima de 1:2, uso de probes via vaginal/anal (perineometria), em sessóes de 15 a 30 minutos. Os protocolos de tratamento ${ }^{27}$ que apresentaram melhora dos sintomas de UrI em até $90 \%$ e cura em até $50 \%$ dos casos utilizavam frequências de 10 a $20 \mathrm{~Hz}$, largura de pulso de $200 \mathrm{~ms}$ com média de duração de 9 a 12 semanas, com aplicação semanal de 2 a 3 vezes por semana. Exceto a modalidade de aplicação em dermátomo sacral ${ }^{23}$, todas as outras formas de eletroestimulação apresentaram evidências de forte associação $(\mathrm{p} \leq 0,001)$ à melhora da HD e redução da UrI resultante ${ }^{20}$. Os estudos que utilizaram a forma de eletroestimulação associada a outros procedimentos como a cinesioterapia ${ }^{26}$ e cinesioterapia com biofeedba$\mathrm{ck}^{15,27}$ demonstraram que os indivíduos apresentavam menos queixas de perda urinária, melhora da força muscular e da resistência e contraçóes mais rápidas do assoalho pélvico $(\mathrm{p} \leq 0,05)$.

O uso do Biofeedback como terapia associada está correlacionado a significante redução nas perdas urinárias. Em estudo ${ }^{15}$ que comparava três protocolos $(\mathrm{P}) \mathrm{de}$ tratamento $(\mathrm{P} 1=$ exercícios do assoalho pélvico; $\mathrm{P} 2=\mathrm{P} 1$ + biofeedback eletromiográfico $(\mathrm{BEmG})$ e; P3 $=\mathrm{P} 1$ + P2 + EENM intravaginal) durante 9 semanas, foi verificado que o grupo de indivíduos submetidos ao $\mathrm{P} 3$ apresentou redução mais significativa de perdas urinárias. Em outro estudo $^{27}$, comparando grupos de indivíduos submetidos a exercícios do assoalho pélvico e BEmG e outro a estas técnicas e EENM, os autores concluíram que associar EENM ao programa convencional de tratamento aumen- 
Quadro 1

Artigos selecionados que apresentam procedimentos fisioterapêuticos para o manejo da hiperatividade do detrusor na Esclerose Múltipla

\begin{tabular}{|c|c|c|c|c|}
\hline ESTUDO & DELINEAMENTO & OBJETIVO & PROCEDIMENTO & RESULTADO \\
\hline Primus $(1992)^{22}$ & $\begin{array}{c}\text { ECA } \\
27 \text { mulheres } \\
3 \mathrm{x} / \text { semana } \\
9 \text { semanas }\end{array}$ & $\begin{array}{l}\text { Avaliar o efeito da } \\
\text { eletroestimulaçáo na } \\
\text { melhora da UrI } \\
\text { resultante da HD }\end{array}$ & $\begin{array}{l}\text { Perineometria vaginal } \\
\left(200 \mathrm{Us}, 20 \mathrm{~Hz}, 15^{\prime}\right)\end{array}$ & $\begin{array}{l}\downarrow \text { perdas urinárias, } \\
\text { melhora da UrI } \\
\quad(\mathrm{p} \leq 0,05)\end{array}$ \\
\hline Vahtera et al $(1997)^{28}$ & $\begin{array}{c}\text { CC } \\
80 \text { indivíduos } \\
6 \text { sessóes } \\
\text { Orientação por } 6 \text { meses }\end{array}$ & $\begin{array}{c}\text { Determinar o efeito da } \\
\text { reabilitaçáo do assoalho } \\
\text { pélvico e orientaçóes } \\
\text { na HD }\end{array}$ & $\begin{aligned} \mathrm{GT}= & \mathrm{EENM}+\text { Kegel }+ \\
& \text { orientaçôes } \\
\mathrm{GC}= & \text { sem intervenção }\end{aligned}$ & $\begin{array}{l}\mathrm{GT}: \downarrow \mathrm{UrI} \\
(\mathrm{p} \leq 0,05)\end{array}$ \\
\hline De Ridder et al $(1999)^{25}$ & $\begin{array}{c}\text { EC } \\
30 \text { mulheres } \\
3 \times / \text { semana } \\
12 \text { semanas }\end{array}$ & $\begin{array}{l}\text { Verificar o efeito da } \\
\text { reabilitaçáo do assoalho } \\
\text { pélvico na HD }\end{array}$ & Kegel & $\begin{array}{c}\uparrow \text { FM e potência } \\
(\mathrm{p}<0,001) \text { e da } \\
\text { capacidade vesical } \\
(\mathrm{p} \leq 0,05)\end{array}$ \\
\hline McClurg et al (2006) ${ }^{15}$ & $\begin{array}{c}\text { ECA } \\
30 \text { mulheres } \\
2 \mathrm{x} / \text { semana } \\
9 \text { semanas }\end{array}$ & $\begin{array}{l}\text { Determinar a efetividade } \\
\text { de um programa de } \\
\text { treinamento do assoalho } \\
\text { pélvico na HD }\end{array}$ & $\begin{array}{c}\text { G1: Kegel } \\
\text { G2: Kegel + BEmG } \\
\text { G3: Kegel + BEmG + } \\
\text { EENM }\end{array}$ & $\begin{array}{c}\text { G1 e G2: } \downarrow \text { HD } \\
(\mathrm{p} \leq 0,05) \\
\text { G3: } \downarrow \text { perdas urinárias } \\
\text { e melhora no Pad Test } \\
\quad(\mathrm{p}=0.001)\end{array}$ \\
\hline Fiorback et al $(2007)^{23}$ & $\begin{array}{c}\text { EC } \\
14 \text { indivíduos } \\
3 \mathrm{x} / \text { semana } \\
12 \text { semanas }\end{array}$ & $\begin{array}{c}\text { Avaliar o efeito da } \\
\text { eletroestimulaçáo no } \\
\text { dermátomo sacral para } \\
\text { HD }\end{array}$ & $\begin{array}{c}\text { EENM } \\
(\text { (200Us, 10Hz. 30') }\end{array}$ & Não há efeito \\
\hline MClurg et al $(2008)^{27}$ & $\begin{array}{c}\text { ECA } \\
74 \text { indivíduos } \\
2 \mathrm{x} / \text { semana } \\
9 \text { semanas }\end{array}$ & $\begin{array}{c}\text { Avaliar o efeito da } \\
\text { reabilitação do assoalho } \\
\text { pélvico na HD }\end{array}$ & $\begin{array}{l}\mathrm{G} 1=\text { Kegel }+\mathrm{BEmG} \\
\mathrm{G} 2=\underset{\mathrm{Kegel}}{\mathrm{EENM}} \mathrm{BEmG}+\end{array}$ & $\begin{array}{c}\text { G1 e G2: Melhora da } \\
\text { UrI }(p \leq 0,05) \\
\text { G2: melhora mais } \\
\text { significativa }(p=0,0028)\end{array}$ \\
\hline Kabay et al $(2008)^{20}$ & $\begin{array}{c}\text { EC } \\
29 \text { indivíduos } \\
2 \mathrm{x} / \text { semana } \\
12 \text { semanas } \\
\end{array}$ & $\begin{array}{c}\text { Determinar o efeito } \\
\text { agudo da estimulação } \\
\text { do nervo tibial posterior } \\
\text { na HD }\end{array}$ & $\begin{array}{c}\text { EENM } \\
\left(200 \mathrm{Us}, 20 \mathrm{~Hz}, 25^{\prime}\right)\end{array}$ & $\downarrow \mathrm{HD}(\mathrm{p} \leq 0,001)$ \\
\hline Kabay et al (2009) ${ }^{21}$ & $\begin{array}{c}\mathrm{EC} \\
19 \text { indivíduos } \\
2 \mathrm{x} / \text { semana } \\
12 \text { semanas } \\
\end{array}$ & $\begin{array}{c}\text { Avaliar o efeito da } \\
\text { estimulaçáo do nervo } \\
\text { tibial posterior na HD }\end{array}$ & $\begin{array}{c}\text { EENM } \\
\left.(200 \mathrm{Us}, 10 \mathrm{~Hz}, 25)^{\prime}\right)\end{array}$ & $\downarrow \mathrm{HD}(\mathrm{p} \leq 0,001)$ \\
\hline Raviv et al $(2009)^{24}$ & $\begin{array}{c}\text { EC } \\
28 \text { indivíduos } \\
\text { Aplicação diária } \\
4 \text { semanas } \\
\end{array}$ & $\begin{array}{c}\text { Avaliar o efeito da } \\
\text { terapia craniosacral na } \\
\text { UrI da HD }\end{array}$ & $\begin{array}{l}\text { Manobras miofasciais em } \\
\text { regiāo crâniosacral }\end{array}$ & $\begin{array}{l}\downarrow \text { volumes residuais } \\
\text { e UrI }(\mathrm{p} \leq 0,05)\end{array}$ \\
\hline Lucio et al $(2010)^{26}$ & $\begin{array}{c}\text { ECA } \\
27 \text { indivíduos } \\
3 \mathrm{x} / \text { semana } \\
12 \text { semanas }\end{array}$ & $\begin{array}{c}\text { Avaliar o efeito do } \\
\text { treinamento do assoalho } \\
\text { pélvico no tratamento } \\
\text { da UrI }\end{array}$ & $\begin{array}{c}\text { G1: Perineometria } \\
\text { vaginal }+ \\
\text { Kegel } \\
\text { G2: Perineometria } \\
\text { vaginal } \\
\left(200 \text { Us, }^{2} 20 \mathrm{~Hz}, 15^{\prime}\right)\end{array}$ & $\begin{array}{c}\mathrm{G} 1: \uparrow \mathrm{FM} \mathrm{e} \\
\text { contraçóes mais rápidas } \\
(\mathrm{p} \leq 0,001)\end{array}$ \\
\hline
\end{tabular}

BEmG: Biofeedback eletromiográfico, CC: caso-controle, EC:estudo coorte, ECA: ensaio clínico aleatorizado, EENM: Eletroestimulação neuromuscular, FM: força muscular, G: grupo, GC: grupo controle, GT: grupo tratamento, HD: hiperatividade do detrusor, Kegel: Exercícios do assoalho pélvico, UrI: urge-incontinência.

tou significativamente os índices de efetividade no tratamento de UrI em indivíduos com EM.

$\mathrm{Na}$ terapia manual, somente um estudo ${ }^{24}$ apresentou resultados significativos $(\mathrm{p}<0.0001)$ para o manejo da HD. Neste, a aplicação isolada e diária da terapia crâ- niossacral foi associada à diminuição dos volumes residuais e sintomas de UrI.

A aplicação da cinesioterapia, com destaque para o protocolo de exercícios de Kegel, foi apresentada em forma combinada com eletroestimulação e Biofeedback ${ }^{15,26-27}$ 
e em um estudo foi a única modalidade terapêutica para o manejo do quadro clínico da $\mathrm{HD}$ na $\mathrm{EM}^{25}$. Em ambas as formas foi verificada melhora da UrI devido ao aumento da força e da resistência do assoalho pélvico. Houve resultados favoráveis ao aumento da força e potência muscular do assoalho pélvico ( $<<0,001)$ e aumento na capacidade vesical $(\mathrm{p} \leq 0,05)$ quando eram realizados apenas exercícios de $\mathrm{Kegel}^{25}$. Combinando perineometria vaginal e exercícios de $\mathrm{Kegel}^{26}$, houve associação fortemente significativa ( $\mathrm{p} \leq 0,001)$ com a melhora do quadro de UrI no grupo de indivíduos submetidos exclusivamente a esta abordagem cinesioterapêutica.

Foi investigado o papel da abordagem utilizando orientações à indivíduos com EM e HD ${ }^{28}$. A amostra deste estudo foi composta por homens e mulheres com EM e HD divididos em grupo controle (sem intervenção) e grupo tratamento (submetido a 6 sessóes de exercícios de Kegel e eletroestimulação e orientados, durante 6 meses, sobre a importância da prática de exercícios de Kegel em ambiente domiciliar). Foi observado que no grupo tratamento o sintoma de UrI foi menos frequente e de forma mais branda que no grupo controle $(\mathrm{p} \leq 0,05)$.

\section{DISCUSSÃO}

O procedimento menos invasivo e menos lesivo deve ser a primeira escolha para tratar os diferentes tipos de disfunções vésico-esfincterianas, como é o caso da $\mathrm{UrI}$ na $\mathrm{HD}^{29}$. A grande vantagem do tratamento medicamentoso é sua praticidade, tanto para o paciente quanto para a equipe de profissionais. Entretanto têm sido muito indicada as intervençóes comportamentais, com destaque para a abordagem fisioterapêutica que pode auxiliar o paciente a reeducar o controle miccional, desenvolver estratégias para minimizar e/ou eliminar as disfunçóes miccionais além de ser mais fisiológica, com poucos efeitos colaterais e contra-indicaçóes, de custo relativamente baixo e que não comprometem tratamentos concomitantes, nem futuros ${ }^{30}$.

Há escassez de estudos com bons níveis de evidência sobre a utilização de procedimentos fisioterapêuticos na $\mathrm{HD}$ associada a $\mathrm{EM}^{18}$. Nesta revisão 16 artigos foram excluídos por não apresentarem dados conclusivos quanto à efetividade das técnicas. Assim, há necessidade da análise criteriosa dos recursos, técnicas e protocolos te- rapêuticos de estudos com delineamentos metodologicamente adequados visando estabelecer um consenso sobre as indicaçôes e efeitos que justifiquem sua aplicação.

A eletroestimulação foi elegível como o recurso mais utilizado tanto na forma isolada como na combinada com resultados significativos na UrI. Ela usualmente é recomendada como terceira linha terapêutica ou para casos excepcionais ${ }^{31}$. O estímulo elétrico promove o fortalecimento da musculatura perineal e melhora na transmissão da pressão abdominal ${ }^{27}$. A UrI é atenuada pela inibição reflexa da contração detrusora ${ }^{32}$.

$\mathrm{Na}$ utilização da eletroestimulação de forma isolada foi evidenciado que há necessidade de uso em longo prazo para a manutenção dos efeitos ${ }^{21}$, embora não seja mensurado este período em nenhum estudo. Estudos em outras populaçóes com queixa de UrI apresentam índices de cura e melhora em até $60 \%$ dos casos com o uso da eletroestimulação ${ }^{33}$. Foi observado que $85 \%$ dos indivíduos ficaram livres do sintoma de UrI após a aplicação de eletroestimulação por 9 semanas contínuas porém após 3 meses da interrupção da técnica apenas 18\% permaneciam sem UrI, embora os que apresentaram recidiva sintomática demonstravam quadro mais leve de UrI em relação ao período pré-aplicação ${ }^{22}$.

A eletroestimulação periférica foi o diferencial nos grupos de tratamento ${ }^{15,27}$. Comparando o efeito de diferentes protocolos na UrI em indivíduos com EM, foi apresentada uma resposta favorável e estatisticamente significante para a redução do tempo de perdas urinárias $(p \leq 0,05)$ quando o protocolo era composto por exercícios pélvicos, BEmG e eletroestimulação perineal em relação aos grupos de indivíduos submetidos aos protocolos compostos apenas por exercícios pélvicos ou exercícios pélvicos associados a BEmG. Entretanto, analisando o efeito da eletroestimulação isolada e aplicada em associação com exercícios de $\mathrm{Kegel}^{26}$, não foi observada melhora significativa com tendência para a eletroestimulação isolada.

O Biofeedback foi apresentado como um recurso bastante utilizado nas formas associadas de terapia no manejo da UrI em indivíduos com $\mathrm{EM}^{15,27}$. Considerando que o assoalho pélvico forma um apoio elástico para o conteúdo pélvico e abdominal, esta técnica estimula os indivíduos quanto ao desenvolvimento de maior sensação de controle e domínio vesical ${ }^{34}$. No tratamento da 
HD, o biofeedback é utilizado para ensinar o paciente a fazer inibição voluntária do detrusor, por contração seletiva dos músculos do assoalho pélvico. Entre as sessóes de treinamento, com repetição dos exercícios e prática, pressupóe-se que os pacientes melhoram não somente a força muscular, mas o controle de respostas que determinam a continência ${ }^{9}$. Em relação à forma de aplicação, apesar da possibilidade de também ser sob a forma pressórica, os estudos analisaram apenas a eletromiográfica. Nesta forma, há um sensor que detecta a atividade elétrica liberada pelos músculos quando se contraem, esse sinal é amplificado e processado de forma visual ou sonora para o paciente ${ }^{35}$.

Têm sido questionado se o Biofeedback é essencial no sucesso do tratamento da UrI em casos de $\mathrm{HD}^{36,37}$. Estes autores relataram estudos que demonstram eficácia dos exercícios de fortalecimento do assoalho pélvico sem biofeedback e outros demonstraram falhas. Diante dos achados desta revisão, em indivíduos com EM, o BEmG é considerado um recurso na abordagem da UrI porém não é o diferencial no tratamento, uma vez que sua eficácia foi observada somente quando aplicado a outros recursos, sem qualquer confirmação de que sua aplicação na forma isolada possa gerar evidências no tratamento da UrI na EM. Este achado corrobora com a revisão sistemática que avaliou o efeito da fisioterapia como tratamento de primeira escolha na HD e concluiu que há fraca evidência da eficácia do biofeedback associado aos exercícios de assoalho pélvico comparado com os exercícios isoladamente a partir de testes clínicos aleatorizados ${ }^{38}$.

Em relação à terapia manual como abordagem fisioterapêutica da HD na EM, sua atuação específica nesta condição é realizada por meio de manobras miofasciais que utilizam alongamentos e compressão dos pontos de gatilhos "trigger points" realizada na região do assoalho pélvico com o propósito de regularização do sistema nervoso autônomo no tratamento da $\mathrm{IU}^{24,39}$. Apesar de haver apenas um estudo ${ }^{24}$ selecionado que aborda esta técnica deve-se considerar que foi realizado por um delineamento de estudo coorte bem delimitado e com acompanhamento. Portanto, mesmo em uma amostra reduzida (28 indivíduos), os efeitos satisfatórios e significantes nos volumes urinários e UrI devem ser considerados como proposta a outras formas de abordagem na terapia manual para o manejo da HD na EM.

A abordagem cinesioterapêutica na UrI tem como princípio as contraçôes voluntárias e repetitivas do assoalho pélvico realizada por meio de exercícios de fortalecimento dos músculos perineais e abdominais aumentando a força muscular e, consequentemente, a continência urinária pelo estímulo da atividade do esfíncter uretral com melhora do tônus ${ }^{40}$. Estudos mostram evidências de que os exercícios para o assoalho pélvico favorecem melhor suporte do colo vesical ${ }^{41}$. Assim, os exercícios são efetivos para a HD e UrI resultante porque reforçam o reflexo de contração do assoalho pélvico, causando inibição da contração do detrusor e levando também a uma diminuição do desejo da micçãa ${ }^{40}$. A hipótese é que os efeitos dos exercícios do assoalho pélvico na HD são mediados por ação reflexa: "reflexo de inibição voluntária da micção"42. Para manutenção da continência é também importante que a atividade do detrusor seja bloqueada quando os músculos do assoalho pélvico se contraem ${ }^{40}$.

Em todos os estudos os exercícios de Kegel foram a única forma de técnica cinesioterapêutica citada. Estes exercícios consistem na contração voluntária da musculatura pubococcígea com o objetivo de impedir a IU, pois melhoram a força muscular do assoalho pélvico, ajudam na resistência uretral e na pressão de fechamento uretral ${ }^{34}$. A reabilitação do assoalho pélvico, por meio destes exercícios tem sido comumente relatada como abordagem terapêutica para UrI no tratamento da HD de indivíduos com EM sem espasticidade da musculatura pélvica $^{26}$. Utilizados como único procedimento fisioterapêutico ${ }^{25}$, os exercícios de Kegel apresentaram associação altamente significante para a melhora do quadro de UrI ( $p<0,001)$. Em sua forma combinada ${ }^{15,26,27}$, os exercícios de Kegel também apresentaram associação significativa à melhora do quadro de HD, além do que, em um estudo ${ }^{26}$ com dois protocolos, um grupo de indivíduos submetido à perineometria vaginal e exercícios de Kegel e outro à aplicação exclusiva de perineometria, foi verificado que a aplicação dos exercícios de Kegel era o diferencial na abordagem da UrI $(p \leq 0,001)$. Este achado permite indicar, nesta revisão, que os exercícios de Kegel são procedimentos confiáveis no manejo da HD na EM e está em consenso com estudos ${ }^{9,40}$ que apresentam estes exercícios como primeira linha do tratamento da UrI. 
Ainda sobre exercícios de Kegel, um aspecto que deve ser considerado quando exercícios físicos são utilizados como forma de tratamento diz respeito ao seu modo de aplicaçấo. Apesar dos resultados expressivos, os estudos analisados não apresentaram um consenso sobre a frequência e tempo de duração das contraçóes destes exercícios, havendo uma variação de 4 a 10 contrações a cada 20 a 30 segundos $^{15,26,27}$. Seria interessante a realização de pesquisas que estabelecessem protocolos mais detalhados dos exercícios de Kegel, assim como os efeitos comparativos dos protocolos quanto a esta forma de aplicação com abordagem específica em EM.

Outro aspecto que deve ser também ressaltado sobre a aplicação dos exercícios em indivíduos com EM é a condição de fadiga. Segundo Nogueira e colaboradores $(2009)^{43}$, a fadiga é o sintoma mais frequente e incapacitante acometendo entre 70 a 90\% dos casos de EM. Considerando que o exercício físico pode aumentar ainda mais a fadiga de uma pessoa com EM, na aplicação dos exercícios de reabilitação do assoalho pélvico deveriam ser preconizados cuidados de prevenção desta condição. Entretanto, entre os estudos analisados, nenhum considerou este fator limitante para o manejo da UrI. Assim, mais uma vez, ressalta-se a importância do estabelecimento de um consenso, entre os protocolos de tratamento da HD na EM, que utilizam os exercícios de Kegel, quanto a frequência, repetiçóes e velocidade de contraçôes musculares, enfatizando também a prevenção da fadiga em indivíduos com EM e que apresentam HD.

Este estudo apresentou um artigo que demonstra evidências significantes para a abordagem por meio da orientação no tratamento da $\mathrm{UrI}^{28}$. Não se pretende, mesmo diante de um resultado significativo segundo enfoque estatístico, excluir o benefício da abordagem por meio da orientação para o tratamento da HD na EM, uma vez que ela é comprovada em outros casos ${ }^{44}$. Entretanto, não se pode hipervalorizar este procedimento uma vez que já era presumível este resultado significativo. A orientação foi realizada em um grupo o qual foi aplicado um programa de exercícios do assoalho pélvico e eletroestimulação enquanto que o grupo controle náo recebeu nenhuma forma de intervenção.

A terapia comportamental envolve mudança do comportamento miccional. Assim, a orientação do pa- ciente proporciona maior conscientização quanto à importância das estratégias preventivas. Esta técnica pode ser efetiva quando o paciente é orientado e motivado com expectativas reais a serem atingidas ${ }^{9}$. A educação do paciente baseia-se no controle cortical da micção. Conhecimento, habilidade motora e intervalo miccional para a aderência ao programa de modificação comportamental são documentados como agindo no controle do desejo miccional $^{44}$.

Dentre os estudos selecionados, 2 artigos ${ }^{15,27}$ citaram o uso combinado de exercícios de Kegel, eletroestimulação e BEmG com evidências de melhora em reduzir os sintomas urinários de UrI. Estes achados estão de acordo com a relação entre a associação e a manutenção dos resultados de melhora da UrI na EM².

Os procedimentos citados com poder estatístico comprovado, viáveis como forma de tratamento para a condição de HD e UrI, são os exercícios de Kegel, a terapia crâniossacral, eletroestimulação nas formas de perineometria vaginal e eletroestimulação do nervo tibial posterior, BEmG e abordagem por meio de orientaçóes sobre a importância da realização de exercícios de Kegel em ambiente domiciliar. Em relação ao tempo de duração, os estudos apresentaram abordagens de 4 semanas a 6 meses e não se pode estabelecer nenhuma correlação entre tempo de tratamento e efetividade da abordagem com esses dados, uma vez que todos apresentaram resultados significativos.

Apesar dos estudos apresentarem evidências estatísticas da aplicabilidade de suas abordagens para HD/ UrI na EM é preciso considerar suas limitaçóes. As amostras eram heterogêneas com grupos variando de 14 a 80 indivíduos, algumas técnicas, quando aplicadas isoladamente, não foram utilizadas associadas a outras e vice-versa para verificar se o seu efeito não estaria oculto ou se potencializaria o efeito de outras técnicas. Em alguns casos, havia previsão de achado estatisticamente significativo devido a erros no delineamento dos estudos. Nenhum estudo analisou a resposta aos procedimentos fisioterapêuticos por gênero. Assim, observa-se que os níveis de evidência e os delineamentos escolhidos são insuficientes para demonstrar se os procedimentos fisioterapêuticos demonstrados neste estudo apresentam resultados aplicáveis clinicamente.

Os estudos selecionados não contemplam todos 
os procedimentos fisioterapêuticos que podem ser utilizados na abordagem da HD. Treinamento vesical com o uso de manobras e técnicas de esvaziamento vesical, esvaziamento vesical incitado e cronometrado, estímulo com biofeedback de pressão, o uso de pessários vaginais, cones vaginais, perineometria anal, acupuntura, eletroacupuntura são outras formas de abordagem que também devem ser pesquisadas nesta disfunção em pacientes com EM. Sugere-se a realização de mais estudos, preferencialmente com delineamento de ensaio clínico aleatorizado que sejam sensíveis, válidos e fidedignos, com tamanho de amostra suficiente e grupos mais homogêneos para esclarecer os efeitos dos procedimentos fisioterapêuticos no manejo da HD (UrI) decorrente da EM, comprovando a eficácia das técnicas.

\section{CONCLUSÃO}

A abordagem combinada dos procedimentos fisioterapêuticos é encorajada no manejo da HD e UrI resultante, sendo proposta a utilização dos exercícios de Kegel e sua orientação como atividade domiciliar, eletroestimulação nas formas de perineometria vaginal e eletroestimulação do nervo tibial posterior, BEmG e terapia craniossacral para o tratamento e manutenção dos resultados a longo prazo. Não há, com base nos resultados apresentados, nenhuma técnica que predomine em termos de benefícios a longo e curto prazo para ser indicada como essencial na abordagem da HD em indivíduos com EM.

\section{REFERÊNCIAS}

1.Rowland L. Merritt Tratado de Neurologia. 10ed. Rio de Janeiro: Guanabara Koogan, 2002, 887p.

2.Zivadinov $\mathrm{R}$. The use of standardized incidence and prevalence rates in epidemiological studies on multiple sclerosis. A meta-analysis study. Neuroepidemiology 2003;22:65-74.

http://dx.doi.org/10.1159/000067107

3.Kalsi V, Fowler CR. Therapy insight: bladder dysfunction associated with multiple sclerosis. Nat Clin Pract Urol 2005;2:492-501.

http://dx.doi.org/10.1038/ncpuro0323

4.Gallien P, Robineau S, Nicolas B, Le Bot MP, Brissot R, Verin M. Vesicourethral dysfunction and urodynamic findings in multiple sclerosis: a study of 149 cases. Arch Phys Med Rehabil 1998;79:255-7.

http://dx.doi.org/10.1016/S0003-9993(98)90003-X

5.Ozyurt C, Çal Ç, Delibas M, Araç N, Sagduyu A. Voiding dysfunction in multiple sclerosis. Braz J Urol 2000;26:315-20.

6.Abrams P, Cardozo L, Fall M, Griffiths D, Rosier P, Ulmsten U, et al. The standardisation of terminology of lower urinary tract function: report from the Standardisation Sub-committee of the International Continence Society. Neurourol Urodyn 2002;21:167-78.

\section{http://dx.doi.org/10.1002/nau.10052}

7.Gandolpho L, Heilberg IP, Cuppoloni MM. Atualização em Infecções Urinárias: Bexiga Neurogênica. São Paulo: Moreira Jr., 1995, 141p.

8.Ouslander JG. Management of overactive bladder. N Engl J Med 2004;350:786-99.

http://dx.doi.org/10.1056/NEJMra032662

9.Mesquita LA, César PM, Monteiro MVC, Silva Filho AL. Terapia comportamental na abordagem primária da hiperatividade do detrusor. Femina 2010;38:23-9.

10.Donzé C, Hautecoeur P. Urinary, sexual, and bowel disorders in early-stage multiple sclerosis. Rev Neurol 2009;165:148-55.

11.Pavan K, Miguez PB, Marangoni BEM, Tilbery CP, Lianza S. Comportamento da incontinência urinária em pacientes com esclerose múltipla e a sua influência na qualidade de vida. Med Reabil 2010;29:1-5.

12.Henze T. Managing Specific symptoms in people with multiple sclerosis. Int MS J 2005;12:60-8.

13. Haslam C. Managing bladder symptoms in people with multiple sclerosis. Nurs Times 2005;101:48-52.

14.Coelho MM. Avaliação urodinâmica na esclerose múltipla. Acta Urol 2009;3:9-14.

15.McClurg D, Ashe RG, Marshall K, Lowe-Strong AS. Comparison of pelvic floor muscle training, electromyography biofeedback, and neuromuscular electrical stimulation for bladder dysfunction in people with multiple sclerosis: a randomized pilot study. Neurourol Urodyn 2006;25:337-48.

http://dx.doi.org/10.1002/nau.20209

16.Hay-Smith J, Herbison P, Ellis G, Moore K. Anticholinergic drugs versus placebo for overactive bladder syndrome in adults. Cochrane Database Syst Rev 2005;(4):CD003781.

17. Wein AJ, Rackley RR. Overactive bladder: a better understanding of pathophysiology, diagnosis and management. J Urol 2006;175(3Pt 2):S5-10.

http://dx.doi.org/10.1016/S0022-5347(05)00313-7

18.Berghmans B. El papel del fisioterapeuta pélvico. Actas Urol Esp 2006;30:110-22.

19.McClurg D, Lowe-Strong A, Ashe R. Pelvic floor training for lower urinary tract dysfunction in MS. Nurs Times 2009;7:45-7.

20.Kabay SC, Yucel M, Kabay S. Acute effect of posterior tibial nerve stimulation on neurogenic detrusor overactivity in patients with multiple sclerosis: urodynamic study. Urology 2008;71:641-5.

http://dx.doi.org/10.1016/j.urology.2007.11.135

21.Kabay S, Kabay SC, Yucel M, Ozden H, Yilmaz Z, Aras O, et al. The clinical and urodynamic results of a 3-month percutaneous posterior tibial nerve stimulation treatment in patients with multiple sclerosis-related neurogenic bladder dysfunction. Neurourol Urodyn 2009;28:964-8.

http://dx.doi.org/10.1002/nau.20733

22.Primus G. Maximal electrical stimulation in neurogenic detrusor hyperactivity: experiences in multiple sclerosis.Eur J Med 1992;1:80-2.

23. Fiorback MV, Van Rey FS, Rijkhoff NJ, Nøhr M, Petersen T, Heesakkers JP. Electrical stimulation of sacral dermatomes in multiple sclerosis patients with neurogenic detrusor overactivity. Neurourol Urodyn 2007;26:525-30.

http://dx.doi.org/10.1002/nau.20363

24.Raviv G, Shefi S, Nizani D, Achiron A. Effect of craniosacral therapy on lower urinary tract signs and symptoms in multiple sclerosis. Complement Ther Clin Pract 2009;15:72-5. 
http://dx.doi.org/10.1016/j.ctcp.2008.12.006

25.DeRidder D, Vermeulen C, Ketelaer P, Van Poppel H, Baert L. Pelvic floor rehabilitation in multiple sclerosis. Acta Neurol Belg 1999;99:61-4.

26.Lúcio AC, Campos RM, Perissinotto MC, Miyaoka R, Damasceno BP, D'ancona CA. Pelvic floor muscle training in the treatment of lower urinary tract dysfunction in women with multiple sclerosis. Neurourol Urodyn 2010;29:1410-3.

http://dx.doi.org/10.1002/nau.20941

27.McClurg D, Ashe RG, Lowe-Strong AS. Neuromuscular electrical stimulation and the treatment of lower urinary tract dysfunction in multiple sclerosis--a double blind, placebo controlled, randomised clinical trial. Neurourol Urodyn 2008;27:231-7.

http://dx.doi.org/10.1002/nau.20486

28.Vahtera T, Haaranen M, Viramo-Koskela AL, Ruutiainen J. Pelvic floor rehabilitation is effective in patients with multiple sclerosis. Clin Rehabil 1997;11:211-9.

http://dx.doi.org/10.1177/026921559701100304

29. Yoshimura N, Chancellor MB. Current and future pharmacological treatment for overactive bladder. J Urol 2002;168:1897-913.

http://dx.doi.org/10.1097/00005392-200211000-00004

http://dx.doi.org/10.1016/S0022-5347(05)64261-9

30.Smith PP, McCrery RJ, Appell RA. Current trends in the evaluation and management of female urinary incontinence. CMAJ 2006;175:1233-40.

http://dx.doi.org/10.1503/cmaj.060034

31. The management of urinary incontinence in women (Endereço da Internet). Inglaterra: National Institute for Health and Clinical Excellence (última atualização em: 10/ 2006; citado em: 01/2009). Disponível em: www.nice.org.uk.

32.Yamanishi T, Kamai T, Yoshida KI. Neuromodulation for the treatment of urinary incontinence. Int J Urol 2008;15:665-672.

http://dx.doi.org/10.1111/j.1442-2042.2008.02080.x

33.Van Balken MR, Vandoninck V, Gisolf KW, Vergunst H, Kiemeney LA, Debruyne FM, et al. Posterior tibial nerve stimulation as neuromodulative treatment of lower urinary dysfunction. J Urol 2001;166:914-18.

http://dx.doi.org/10.1097/00005392-200109000-00025

http://dx.doi.org/10.1016/S0022-5347(05)65863-6

34.Vapnek JM. Urinary Incontinence: Screening and Treatment of Urinary
Dysfunction. Geriatrics 2001;56,10:25-32.

35.Magaldi C, Araújo RC, Pacetta AM. Fisioterapia Uroginecológica: Incontinência Urinária e Prolapso. O COFFITO 2002; 28-32

36.Diokno AC, Sampselle CM, Herzog AR, Raghunathan TE, Hines S, Messer KL, et al. Prevention of urinary incontinence by behavioral modification program: a randomized, controlled trial among older women in the community. J Urol 2004;171:1165-71.

http://dx.doi.org/10.1097/01.ju.0000111503.73803.c2

37. Wallace SA, Roe B, Williams K, Palmer M. Bladder training for urinary incontinence in adults. Cochrane Database of Systematic Reviews 2004, Issue 1. Art. No.: CD001308. DOI: 10.1002/14651858.CD001308.pub2.

http://dx.doi.org/10.1002/14651858.CD001308.pub2

38.Berghmans LCM, Hendriks HJ, de Bie RA, van Waalwijk, van Doorn ES, Bo $\mathrm{K}$, et al. Conservative treatment of urge urinary incontinence in women: a systematic review of randomized clinical trials. BJU Int 2000;85:254-63.

http://dx.doi.org/10.1046/j.1464-410x.2000.00434.x

39. Weiss JM. Pelvic floor myofascial trigger points: manual therapy for interstitial cystitis and the urgency-frequency syndrome. J Urol 2001;166:2226-31. http://dx.doi.org/10.1016/S0022-5347(05)65539-5

40.Hay-Smith J, Dumoulin C. Pelvic floor muscle training versus no treatment, or inactive control treatments, for urinary incontinence in women (Cochrane Review). In: The Cochrane Library, Oxford: Update Software, Issue 3, 2008.

41.Messer KL, Hines SH, Raghunathan TE, Seng JS, Diokno AC, Sampselle CM. Self-efficacy as a predictor to PFMT adherence in a prevention of urinary incontinence clinical trial. Health Educ Behav 2007;34:942-52.

http://dx.doi.org/10.1177/1090198106295399

42.Shafik A, Shafik IA. Overactive bladder inhibition in response to pelvic floor muscle exercises. World J Urol 2003;20:374-7.

43. Nogueira LC, Nóbrega FR, Lopes KN, Thuler LCS, Papais-Alvarenga RM. The effect of functional limitations and fatigue on the quality of life in people with multiplesclerosis. Arq Neuropsiquiatr 2009;67:812-17.

http://dx.doi.org/10.1590/S0004-282X2009000500006

44.Sampselle CM, Messer KL, Seng JS, Raghunathan TE, Hines SH, Diokno AC. Learning outcomes of a group behavioral modification program to prevent urinary incontinence. Int Urogynecol J Pelvic Floor Dysfunct 2005;16:441-6. http://dx.doi.org/10.1007/s00192-005-1284-7 